\title{
Review: Stimulant Augmentation with Antidepressant in Elderly Patients with Major Depressive Disorder
}

\author{
*Dr. Eva R. Haddad ${ }^{1}$, Dr. Muath F. Marashdeh², Alia I. Qamar ${ }^{3}$, Dr. Malik M. Al-Alwan MD ${ }^{4}$, Dr. \\ Arafat K. Al-Zubi MD \\ ${ }^{1}$ Pharmacist, RMS, \\ ${ }^{2}$ MD, Psychiatry, RMS, \\ ${ }^{3}$ Speech \& Clinical psychotherapist, Jordanian Royal Medical Services, \\ ${ }^{4}$ Psychiatry, Jordanian Royal Medical Services
}

\begin{abstract}
"According to the Centers for Disease Control and Prevention (CDC), depression affects about $1 \%-5 \%$ of the general elderly population, $13.5 \%$ in elderly who require home healthcare, and $11.5 \%$ in older hospital patients" CDC, 2017. Depression in the elderly is accompanied by functional weakening and early death due to suicide and other causes, symptoms may include sadness; lack of interest, power, and ability to concentrate; insomnia and decreased appetite; and co-disease. Elderly patients have a lower response to antidepressant medications than adult patients.
\end{abstract}

\section{Objective}

This study aims to explore the potential of using stimulant augmentation to accelerate and enhance antidepressant response in elderly patients with major depressive disorder.

Data Sources: PubMed, CINAHL, UpToDate, Google Scholar, and relevant English-language literature from 1995-2019 were searched. Keywords were stimulant, augmentation, geriatric, late-life, elderly, depression, and major depressive disorder.

Keywords: Depression in elderly, SSRIs, Resentence, Stimulant augmentation.

Study Selection: The search was limited to elderly adults (generally age 65 years or older) with major depressive disorder and stimulants were used in augmentation of antidepressants. Studies in adult patients and bipolar depressions were excluded.

Results: A total of 50 articles were identified, and 12 studies met inclusion criteria. Four of them were double-blind, placebo-controlled (DBPC) trials and eight were retrospective reviews or open studies. Almost all the studies that evaluated geriatric depression used validated scales, (i.e.: the Hamilton Depression Rating Scale, Montgomery-Asberg Depression Rating Scale). Seven studies discuss the Methylphenidate augmentation of citalopram, while five studies discussed augmentation with other medications in addition to a stimulant. Treatment with citalopram and methylphenidate in combination appears to be a useful strategy for increasing and improving antidepressant response in elderly depressed patients, with a higher rate of remission. The patient's tolerability and safety should be considered. Misperception, nervousness, anxiety, hypomania, and delusions were common side effects in augmentation. Few cardiovascular adverse events were observed, thus this combination should be avoided in patients with coronary heart disease (CHD) and arrhythmias. Cognitive functioning showed an improvement with stimulant augmentation, but methylphenidate use did not add any cognitive improvement. 
Conclusion: Stimulant augmentation with the antidepressants in elderly patients with major depressive disorder was found to be a statistically and clinically significant treatment strategy. 